\title{
Kinematic Analysis of the Cervical Cord and Cervical Canal by Dynamic Neck Motion
}

\author{
Kenji Endo, Hidekazu Suzuki, Hirosuke Nishimura, Hidetoshi Tanaka, \\ Takaaki Shishido, Kengo Yamamoto \\ Department of Orthopedic Surgery, Tokyo Medical University, Tokyo, Japan
}

\begin{abstract}
Study Design: Normal cervical sagittal length patterns were measured by magnetic resonance imaging (MRI).
Purpose: The aim of this study was to evaluate the relationship of sagittal length patterns between the cervical cord and the cervical canal in flexion-extension kinematics.

Overview of Literature: Cervical dynamic factors sometimes cause a cervical spondylotic myelopathy in elderly subjects and an overstretching myelopathy in juvenile subjects. Previous studies showed the length changing of the cervical cord in flexion and extension. However, there is no detailed literature about the relationship between cervical vertebral motion and cord distortion yet.

Methods: Sixty-two normal subjects ( 28 male and 34 female, $42.1 \pm 8.5$ years old) without neck motion disturbances and abnormalities on cervical $\mathrm{X}$-ray and MRI were enrolled in this study.

Results: The cervical cord length was significantly longer in flexion and significantly shorter in extension in all cervical cord sagittal lines. The cervical canal length pattern was also the same as the cervical cord. The elongation of the cervical cord and canal was the largest at the site of the posterior cervical canal and the shortest at the anterior canal site. The positions of the cerebellar tonsils were verified at each neck position.

Conclusions: The posterior elements of the cervical canal were most affected by neck motion. Movement directions of the upper cervical cord were verified among the various neck positions.
\end{abstract}

Keywords: Cervical spine; Biomechanics; Magnetic resonance imaging

\section{Introduction}

The cervical spine normally has the greatest sagittal motion within the total spine and its dynamic factors sometimes cause cervical spondylotic myelopathy in elderly persons [1-3] and overstretching myelopathy in juvenile persons [4]. In 1966, Breig and el-Nadi [5] reported in a cadaveric study that the spinal canal decreases in length when the spine is extended and increases in length when the spine is flexed. The spinal cord follows the changes of length in the spinal canal between flexion and extension. Previous reports also described the length pattern of the cervical cord and sometimes the length pattern of the cervical canal by performing magnetic resonance imaging (MRI) [6-10]. However, the sagittal length pattern is not clear regarding the total cervical vertebral motion. The purpose of this study was to assess the relationship of the cervical length patterns in flexion and extension and to estimate the correlations with the cervical cord and cervical vertebral motion.

Received Jan 17, 2014; Revised Apr 23, 2014; Accepted May 1, 2014

Corresponding author: Kenji Endo

Department of Orthopedic Surgery, Tokyo Medical University,

6-7-1 Nishishinjuku, Shinjuku-ku, Tokyo 160-0023, Japan

Tel: +81-3-3342-6111, Fax: +81-3-3342-5295, E-mail: kendo@tokyo-med.ac.jp 


\section{Materials and Methods}

We enrolled 62 normal subjects (28 male and 34 female, $42.1 \pm 8.5$ years of age) without neck motion disturbances and abnormalities on cervical X-ray and MRI. All subjects provided written informed consent after explanation of the experimental protocol. This study was approved by the Institutional Review Board of our institution.

The measurements of the cervical spine in flexion, neutrality and extension were performed with the patient in the supine position. Cervical flexion was initiated by placing a $20 \mathrm{~cm}$ pillow below the head. Cervical extension was initiated by placing the pillow below the upper thoracic vertebrae. The imaging procedure was as follows: a spine echo sequence with TR $100 \mathrm{~ms}$ and TE $35 \mathrm{~ms}$ was used. The data were displayed on a $128 \times 128$ matrix. After a sagittal scan, an axial scan was performed at the level of the grounded plate $\mathrm{C} 7$ and at the level of the foramen magnum, permitting the exact determination of the midline. One pixel was equal to $0.94 \mathrm{~mm}$ on the sagittal image. On the mid-sagittal image, the length of the cervical cord was measured at the middle from the caudal end of the medulla to the caudal end of the C7 vertebra. On scans, the length of the cervical cord was defined as the length between a line at the caudal side of the pons to the continuation of the line at the lower endplate of C7. The length of the anterior cervical canal was defined as the length through the posterior vertebral line between the top of the dens and the lower end of the C7 vertebra. The length of the posterior cervical canal was defined as the length between the top of the $\mathrm{C} 1$ posterior arch and the lower border of the C7 lamina (Fig. 1A). In order to investigate the upper cervical movement during neck motion, we measured the length between the cerebellar tonsils and the foramen magnum. The position of the cerebellar tonsils was also defined as the length between a line that crosses the caudal end of the cerebellar tonsils and a line that crosses the cord at the foramen magnum (Fig. 1B). The radiographs were measured twice by the first observer (K.E., a board-certified orthopedic spinal surgeon) and then independently measured on different days by a second observer (H.S., also a board-certified orthopedic spinal surgeon). Intra and interobserver agreements for the measurements were made with results
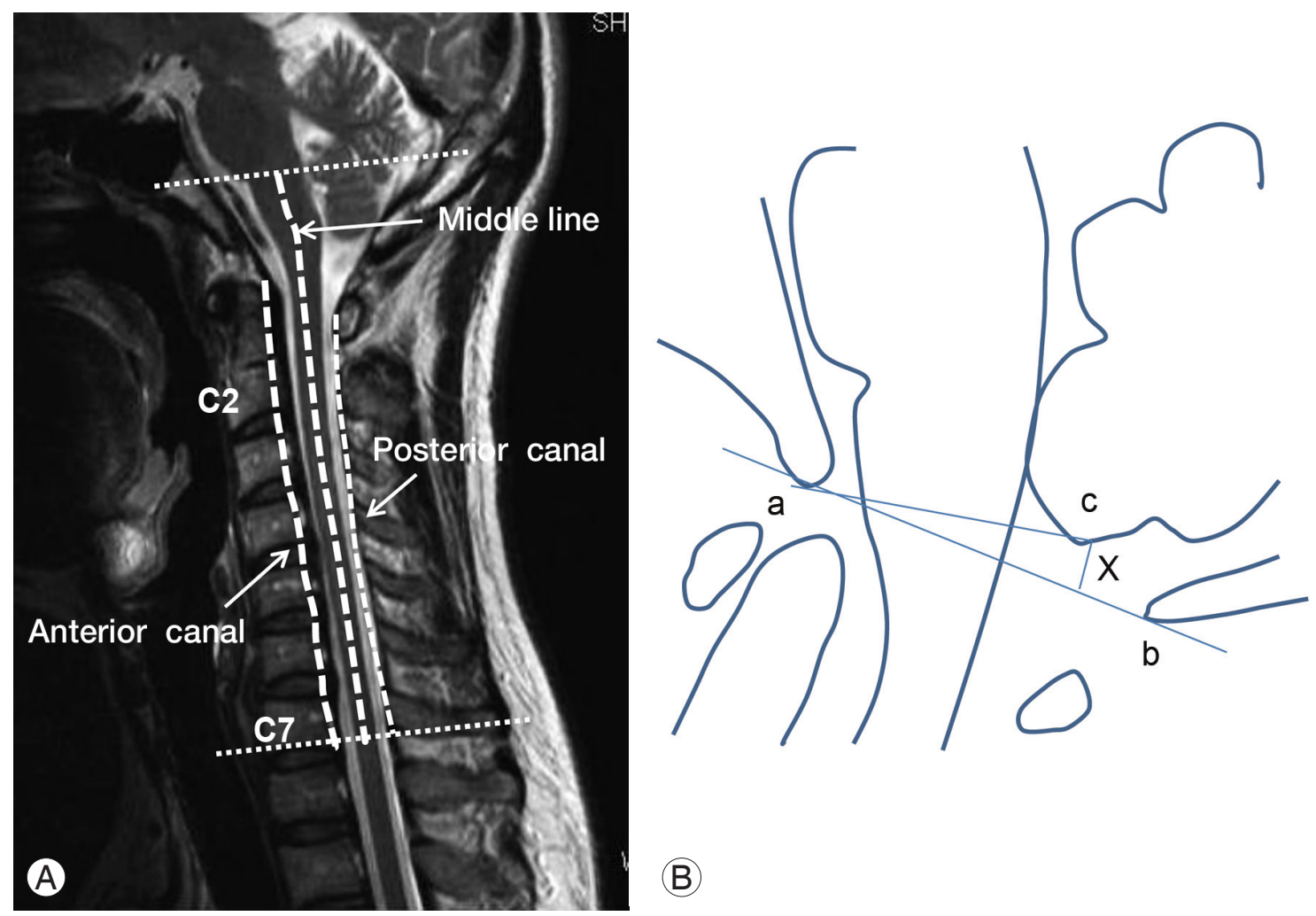

Fig. 1. (A) The lengths of the cervical cord and cervical canal and techniques of measurement of the length of the cervical cord at the anterior, middle and posterior lines are illustrated. $(B)$ The length $(X)$ between the foramen magnum (a-b) and the cerebellar tonsils (c). 
in a reasonable agreement (intraclass correlaton coefficient, 0.98; 95\% confidence interval, 0.98-1.00).

Data were expressed as mean \pm standard deviation. The measurements were tabulated and analyzed using JMP ver. 8.0 (SAS Institute Inc., Cary, NC, USA). The analysis of variance test was used to analyze parametric data. Differences between groups were examined for statistical significance using the Mann-Whitney test. $p$-values less than 0.05 were considered to indicate a statistically significant difference.

\section{Results}

There were significant differences in the sagittal length patterns of the cervical cord and posterior cervical canal vertebrae in neck flexion, neutrality and extension (cervical range of motion, $41^{\circ} \pm 4.5^{\circ}$ ). The lengths of the cervical cord and posterior canal were significantly longer in flexion and significantly shorter in extension (Fig. 2). The elongation of the cervical cord and vertebrae was the largest at the posterior cervical canal line and the shortest at the anterior cervical canal line. The positions of the cerebellar tonsils from the foramen magnum were $6.4 \pm 4.6$ $\mathrm{mm}$ in flexion, $5.5 \pm 4.1 \mathrm{~mm}$ in the neutral position and $5.5 \pm 3.5 \mathrm{~mm}$ in extension. There were no significant differences in the positions of the cerebellar tonsils among the neck positions. Regarding the directions of movement of the cerebellar tonsils, 34 of 60 were ascending, 22 of 60 were descending and 4 of 60 were at the same level in neck flexion from the extension position. Positions of cerebellar tonsils were verified at all neck positions.

\section{Case presentation}

We present the normal case of a 25-year-old man. The length of its cervical cord was the longest in flexion and

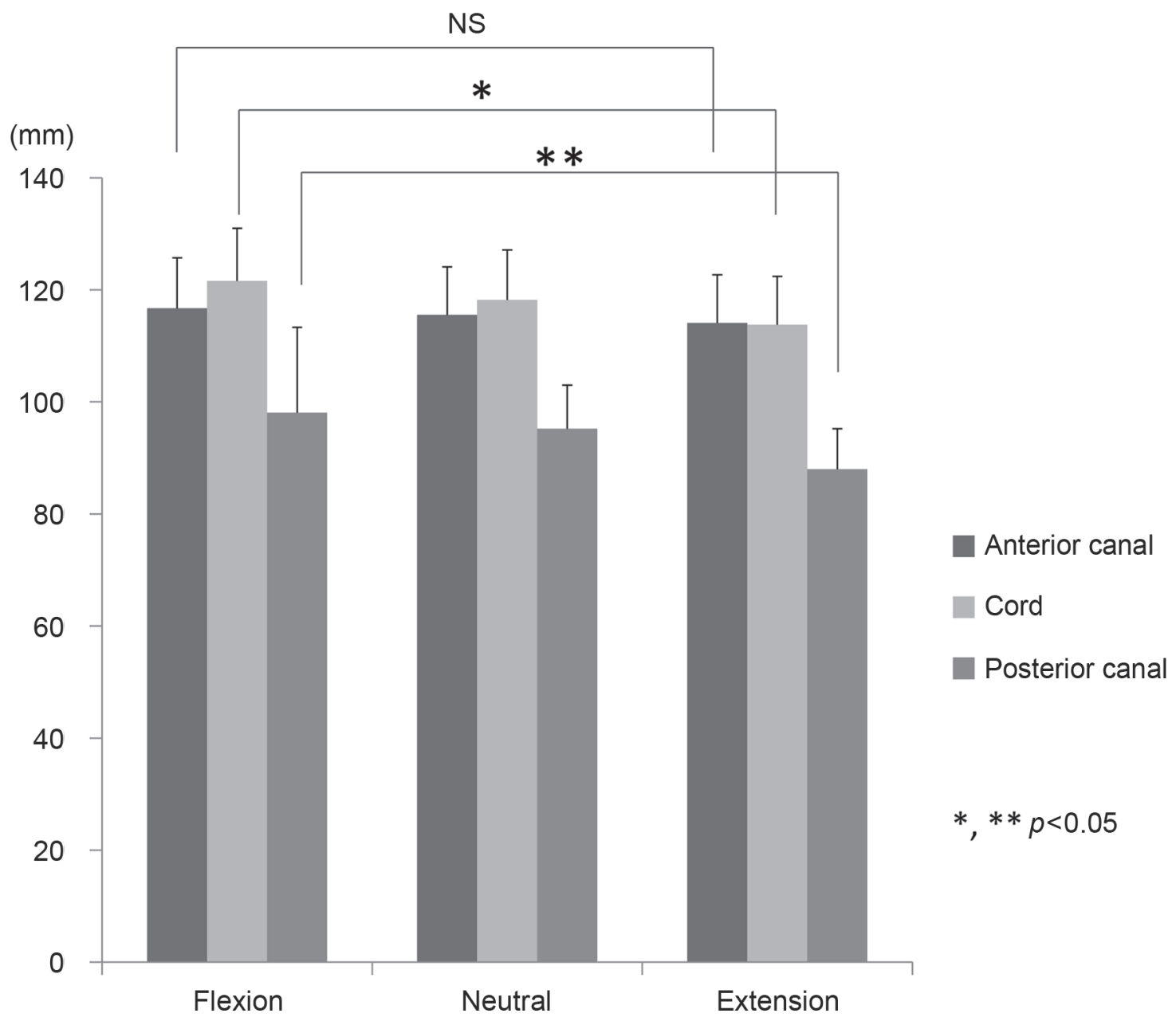

Fig. 2. The lengths of the cervical cord and canal in flexion, neutral position and in extension. NS, not significant. 
the elongation change was the largest in the posterior cervical spinal canal (Fig. 3).

\section{Discussion}

The spinal cord follows the changes in length by the physiological motion of the spinal canal. This occurs by the mechanisms of unfolding, folding and elastic deformation of the spinal cord [11-13].

Breig and el-Nadi [5] performed studies on human cadavers and showed that the spinal canal decreased in length by 8 to $10 \mathrm{~mm}$ when the cervical spine was extended and increased in length when the cervical spine was flexed. The proposed mechanism was that the spinal cord folds like an accordion during extension and unfolds like an accordion during flexion $[8,11,13]$. Kuwazawa et al. [8] reported in their MRI study for the cervical cord at the middle line a mean elongation of the length of the cervical cord from extension to flexion of $11.7 \mathrm{~mm}$ in the recumbent series and of $9.5 \mathrm{~mm}$ in the erect series. However, when taking measurements using the landmarks of cervical vertebrae, it was possible to include the distance of cervical cord shifting accompanied with the cervical canal motion.

In this study, we measured from the caudal side of the pons to minimize an inclusion of the sliding distance. Our resulting in vivo measurement data of the cervical spinal cord showed slightly shorter results than previous data. The mean lengthening was $7.8 \mathrm{~mm}$ in the middle line of the cervical cord; the cervical cord might have been sliding in the rostral and caudal directions from extension to flexion.

Regarding the changes in the cervical canal distance, the length of the cervical cord in flexion was the longest and the length of the cervical cord in extension was the shortest at the anterior, middle and posterior lines. This means that the posterior elements are more mobile than the anterior elements in the cervical canal and that the cervical cord was most elongated in flexion. According to a previous study, the cerebral spinal fluid pressure in patients with Chiari I malformation is increased in neck flexion [14]. That result is similar to the cervical flexion myelopathy in younger subjects [15]. On the other hand, the cervical spondylotic myelopathy in elderly subjects is worse in neck extension due to the dynamic pincer mechanism of the posterior cervical canal elements [16]. The posterior cervical laminoplasty and decompression have yielded good postoperative long term results even if there is an anterior compression such as in the cervical ossification of the posterior longitudinal ligament [17]. When a cervical lordosis is maintained during neck motion, laminectomy and laminoplasty can be considered as resulting in not only the decompression of the cervical cord due to the removal of the posterior elements, but also resulting in improvements in the cervical pathological dynamic factors in elderly persons.

This study had some limitations. It is known that the segmental range of motion changes depending on the
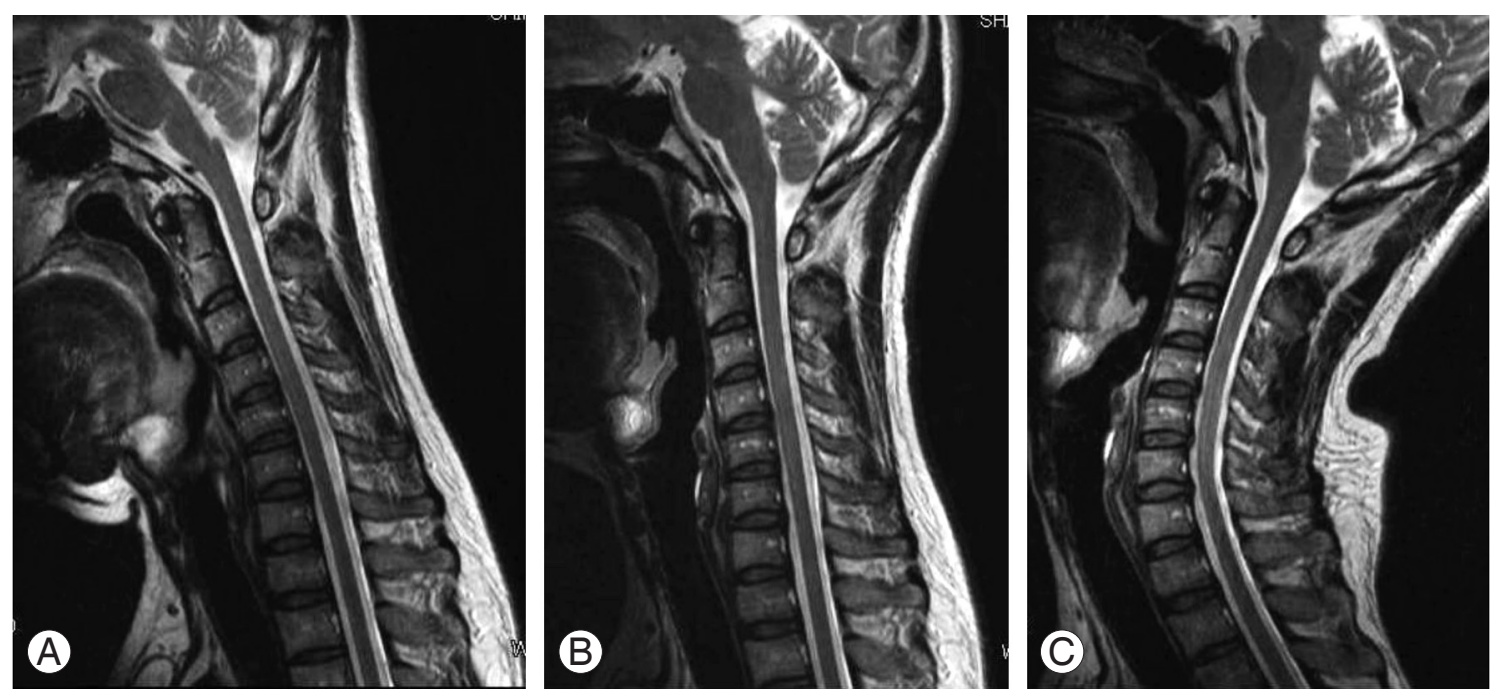

Fig. 3. The cervical magnetic resonance imaging shows (A) the cervical flexion, (B) cervical neutral position and (C) cervical extension. 
cervical curves [18]. This type of research should consider the differences in the original cervical curve. In cervical flexion and extension, the precise measurement of cervical length is difficult in vivo because the cervical cord may move in both the rostral and caudal directions. Despite these limitations, we consider the current results obtained from MRI measurements using clearly defined landmarks could contribute to the estimation of the dynamic effects in cervical motion. In future studies, it may be useful to analyze the relationships between neck motion and cervical sagittal alignment.

\section{Conclusions}

The length of the cervical cord is longest in flexion and shortest in extension. The elongation change was largest in the posterior cervical spinal canal. In terms of length pattern, the posterior elements of the cervical canal were most affected by neck motion. The directions of movement of the upper cervical cord were verified among the various neck positions.

\section{Conflict of Interest}

No potential conflict of interest relevant to this article was reported.

\section{Acknowledgments}

We are indebted to the Department of International Medical Communications of Tokyo Medical University for the editorial review of the English manuscript. The authors would also like to thank Ms. Yuri Amamizu of the Department of Orthopedic Surgery for preparation of the initial English manuscript.

\section{References}

1. Bernhardt M, Hynes RA, Blume HW, White AA 3rd. Cervical spondylotic myelopathy. J Bone Joint Surg Am 1993;75:119-28.

2. Brain WR, Northfield D, Wilkinson M. The neurological manifestations of cervical spondylosis. Brain 1952;75:187-225.

3. Gooding MR. Pathogenesis of myelopathy in cervical spondylosis. Lancet 1974;2:1180-1.
4. Kohno M, Takahashi H, Yagishita A, Tanabe H. "Disproportion theory" of the cervical spine and spinal cord in patients with juvenile cervical flexion myelopathy. A study comparing cervical magnetic resonance images with those of normal controls. Surg Neurol 1998;50:421-30.

5. Breig A, el-Nadi AF. Biomechanics of the cervical spinal cord. Relief of contact pressure on and overstretching of the spinal cord. Acta Radiol Diagn (Stockh) 1966;4:602-24.

6. Bilston LE, Thibault LE. The mechanical properties of the human cervical spinal cord in vitro. Ann Biomed Eng 1996;24:67-74.

7. Kimura S, Hesselink JR, Garfin SR, Kawaji Y, Hasegawa K, Hargens AR. Axial load-dependent cervical spinal alterations during simulated upright posture: a comparison of healthy controls and patients with cervical degenerative disease. J Neurosurg Spine 2005;2:137-44.

8. Kuwazawa Y, Pope MH, Bashir W, Takahashi K, Smith FW. The length of the cervical cord: effects of postural changes in healthy volunteers using positional magnetic resonance imaging. Spine (Phila $\mathrm{Pa}$ 1976) 2006;31:E579-83.

9. Margulies SS, Meaney DF, Bilston LB, Thibault LE, Campeau NG, Riederer SJ. In vivo motion of the human cervical spinal cord in extension and flexion. Proceedings of the 1992 International International Research Council on the Biomechanics of Impact Conference on the Biomechanics of Impact; 1992 Sep. 9-11; Verona, Italy.

10. Muhle C, Wiskirchen J, Weinert D, et al. Biomechanical aspects of the subarachnoid space and cervical cord in healthy individuals examined with kinematic magnetic resonance imaging. Spine (Phila Pa 1976) 1998;23:556-67.

11. Panjabi M, White A 3rd. Biomechanics of nonacute cervical spinal cord trauma. Spine (Phila Pa 1976) 1988;13:838-42.

12. White AA 3rd, Panjabi MM. Biomechanical considerations in the surgical management of cervical spondylotic myelopathy. Spine (Phila Pa 1976) 1988;13: 856-60.

13. White AA, Panjabi MM. Clinical biomechanics of the spine. 2nd ed. Philadelphia: Lippincott; 1990.

14. Tachibana S, Iida H, Yada K. Significance of positive Queckenstedt test in patients with syringomyelia as- 
sociated with Arnold-Chiari malformations. J Neurosurg 1992;76:67-71.

15. Kohno M, Takahashi H, Ide K, Yamakawa K, Saitoh T, Inoue K. Surgical treatment for patients with cervical flexion myelopathy. J Neurosurg 1999;91:33-42.

16. Penning L. Some aspects of plain radiography of the cervical spine in chronic myelopathy. Neurology 1962;12:513-9.
17. Chiba K, Ogawa Y, Ishii K, et al. Long-term results of expansive open-door laminoplasty for cervical myelopathy--average 14-year follow-up study. Spine (Phila Pa 1976) 2006;31:2998-3005.

18. Takeshima T, Omokawa S, Takaoka T, Araki M, Ueda Y, Takakura Y. Sagittal alignment of cervical flexion and extension: lateral radiographic analysis. Spine (Phila Pa 1976) 2002;27:E348-55. 